Pesq. Vet. Bras. 35(3):304-310, março 2015

DOI: $10.1590 / \mathrm{S} 0100-736 \mathrm{X} 2015000300015$

\title{
Estudo endócrino reprodutivo e do comportamento sócio-sexual de sagui-de-tufo-preto (Callithrix penicillata) mantido em cativeiro ${ }^{1}$
}

\author{
Manuela Gonçalves Fraga Geronymo Sgai ${ }^{2 *}$, Cristiane Schilbach Pizzutto ${ }^{2}$, Priscila \\ Viau $^{3}$, Cláudio Alvarenga de Oliveira ${ }^{3}$ e Marcelo Alcindo de Barros Vaz Guimarães ${ }^{3}$
}

\begin{abstract}
Sgai M.G.F.G., Pizzutto C.S., Viau P., Oliveira C.A. \& Guimarães M.A.B.V. 2015. [Reproductive endocrine and socio-sexual behavior study of black-tufted-marmoset (Callithrix penicillata) kept in captivity.] Estudo endócrino reprodutivo e do comportamento sócio-sexual de sagui-de-tufo-preto (Callithrix penicillata) mantido em cativeiro. Pesquisa Veterinária Brasileira 35(3):304-310. Departamento de Reprodução Animal, Faculdade de Medicina Veterinária e Zootecnia, Universidade de São Paulo, Av. Prof. Dr. Orlando Marques de Paiva 87, Cidade Universitária, São Paulo, SP 055508-270, Brazil. E-mail: manuelafraga@hotmail.com

The communication of the female reproductive status in Callithrichidae relies mainly on the socio-sexual behavior, as generally the ovulation is concealed in this primate family by a primary signaling system. In this study the socio-sexual behavior patterns was analyzed in association with the concentration of fecal metabolites of sex steroid hormones progesterone (MFP), estradiol (MFE) and testosterone (MFT) in captive couples of Black-Tufted-Marmoset (Callithrix penicillata), during the different phases of the ovarian cycle. The studied group was composed of four adult couples kept in the São Paulo City Wild Animals Rehabilitation Center. The behavioral patterns were record by focal samplings, with 30 seconds intervals for each observation, five days a week, totalizing 14.400 registers per animal. The measurement of fecal metabolites of progesterone (MFP), estradiol (MFE) and testosterone (MFT) proceeded by enzyme immune assay (EIA). The results allowed to determine the duration of the ovarian cycle and to characterize three different phases (follicular, periovulatory and luteal). It was possible to determine 31 complete cycles that lasted $24.3 \pm 4.1$ days (Mean \pm SD). The follicular and luteal phases lasted $13.04 \pm 4.8$ and the luteal phase $11.2 \pm 4.2$ days. The behavioral patterns (scent marking, sniff genitals, grooming and sexual presentation) were more prevalent in the periovulatory phase as the behavioral variable "proximity" as well. There were no variations in the concentration of MFT in the males during the period studied. The associated analyses of the fecal metabolite of sex steroids and the socio-sexual behaviors led to a better understanding of the factors involved in the reproduction of $C$. penicillata.
\end{abstract}

INDEX-TERMS: Black-tufted-marmoset, Callithrix penicillata, socio-sexual behavior, sex steroids, reproduction.

RESUMO.- A comunicação do estado reprodutivo nos primatas da família Callithrichidae, depende principalmente dos comportamentos sócio-sexuais como um sistema de

\footnotetext{
${ }^{1}$ Recebido em 26 de junho de 2014.

Aceito para publicação em 10 de março de 2015.

${ }^{2}$ Shape Brasil, Rua Dr. Miranda de Azevedo 779, Apto 104, São Paulo, SP 05027-000, Brasil.*Autor para correspondência: manuelafraga@hotmail.com

${ }^{3}$ Departamento de Reprodução Animal, Faculdade de Medicina Veterinária e Zootecnia (FMVZ), Universidade de São Paulo (USP), Av. Prof. Dr. Orlando Marques de Paiva 87, Cidade Universitária, São Paulo, SP 05508270. E-mail: mabvg@usp.br
}

sinalização primário, uma vez que nestas espécies a ovulação não é percebida pelos machos. Neste trabalho, os padrões de comportamentos sócio-sexuais foram analisados em conjunto com as concentrações de metabólitos fecais dos esteróides sexuais progesterona (MFP), estradiol (MFE) e testosterona (MFT) em casais cativos de Sagüi-de-tufos-pretos (Callithrix penicillata), nas diferentes fases do ciclo ovariano. 0 grupo estudado era composto por quarto casais adultos, mantidos no Centro de Reabilitação de Animais Selvagens da prefeitura de São Paulo. Os padrões comportamentais foram registrados pelo método de amos- 
tragem focal por intervalo de tempo a cada 30 segundos, cinco vezes por semana, totalizando 14.400 registros por animal. A mensuração das concentrações de metabólitos fecais dos esteroides sexuais foram realizados pelo método de enzima imunoensaio (EIE). Os resultados obtidos dessas concentrações possibilitaram a determinação endócrina das fases do ciclo ovariano (folicular e luteal) e de suas respectivas durações, assim como a determinação da fase periovulatória. Foram caracterizados 31 ciclos ovarianos

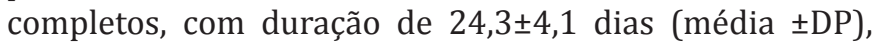
sendo que a fase folicular compreendeu $13,04 \pm 4,8$ dias e a fase lútea $11,2 \pm 4,2$ dias. Os comportamentos sócio-sexuais (marcação por cheiro, cheirar genitália, catação e apresentação sexual) e a variável "proximidade" mostraram-se significativamente mais prevalentes na fase periovulatória do que nas demais fases do ciclo. Não houve alteração das concentrações de MFT dos machos ao longo de todo o período estudado. A análise conjunta das concentrações de metabólitos fecais de esteróides sexuais e dos comportamentos sócio-sexuais possibilitou um melhor entendimento das relações endócrino-comportamentais e reprodutivas de $C$. penicillata.

TERMOS DE INDEXAÇÃO: Sagui-de-tufos-pretos, Callithrix penicillata, comportamentos sócio-sexuais, hormônios esteroides sexuais, reprodução.

\section{INTRODUÇÃO}

Callithrix penicillata, membro da família Callitrichidae, também conhecido como "black pincelled marmoset", (Stevenson \& Rylands 1988, Faria 1989) é nativo de florestas e matas de galerias do cerrado e possui ampla distribuição na natureza. A fragmentação do ambiente e a captura para a venda como animal de estimação tem provocado diminuição populacional de diversas espécies da família Callithrichidae, aumentando o risco de extinção de algumas delas (Beck et al 1982, Carroll 1982). Assim sendo, o sucesso reprodutivo em cativeiro pode ser um importante aliado na manutenção de populações geneticamente saudáveis. 0 sucesso reprodutivo de uma espécie caracteriza-se pela a ocorrência de cópulas, gestação e a sobrevivência da prole, que alcançando a idade adulta, também se reproduza (Lindburg et al. 1986). No estudo comportamental das relações sócio sexuais entre os primatas, a proceptividade, a atratividade e a receptividade caracterizam padrões de comportamento que possibilitam uma análise mais precisa das estratégias reprodutivas de cada espécie (Dixson 2013). A comunicação do momento do evento fisiológico da ovulação e a coordenação do comportamento sexual são importantes para garantir a fertilização bem sucedida (Ziegler 2013).

Em algumas espécies, as fêmeas são capazes de sinalizar sua fertilidade para os machos por meio de sinais múltiplos (Higham et al. 2012), sendo estes muito variáveis entre os primatas (Fedigan 1982, Dixson 1990). Diferentes posturas corporais, gestos, expressões faciais, vocalizações, assim como variações cromáticas e morfológicas de pele podem refletir alterações nas concentrações de estrógenos e progesterona (Carosi et al. 1999, Dixson 2013).

A questão envolvendo a percepção pelo macho do "sta- tus" reprodutivo da fêmea na família Callitrichidae é constantemente debatida no meio científico, sendo que sinalizadores visuais da ovulação, como a coloração da genitália ou intumescimentos perineais não são descritos (Ziegler et al. 1993) e as cópulas podem ocorrer durante todas as fases do ciclo ovariano, inclusive no período gestacional (Dixson 2013).

Técnicas de mensuração hormonal utilizando métodos não-invasivos são consideradas ferramentas essenciais para possibilitar a análise conjunta dos padrões comportamentais observados com os perfis das variações das concentrações de hormônios sexuais (Rimbach et al. 2013).

A análise conjunta dos padrões comportamentais sócio-sexuais e das variações das concentrações dos metabólitos fecais de hormônios ligados à reprodução, possibilitam uma melhor compreensão da biologia reprodutiva gerando as bases para o sucesso reprodutivo da espécie em cativeiro. Na literatura, inexistem informações sobre as características endócrino-reprodutivas ou comportamentais sócio sexuais do C. penicillata, o que ressalta a relevância deste trabalho.

O objetivo desta pesquisa foi descrever e analisar os comportamentos sócio-sexuais e reprodutivos e suas respectivas associações com as concentrações de metabólitos fecais de esteróides sexuais em machos e fêmeas de sagui-de-tufo-preto (Callithrix penicillata), nas diferentes fases do ciclo ovariano.

\section{MATERIAL E MÉTODOS}

\section{Animais}

Foram estudados 8 indivíduos adultos (4 casais) da espécie sagui-de-tufo-preto (Callithrix penicillata), mantidos no Centro de Reabilitação de Animais Silvestres (CRAS) do Departamento de Parques e Áreas Verdes (DEPAVE III) do município de São Paulo, Brasil.

Os animais estavam alocados em casais (Quadro 1), mantidos em gaiolas com ponto de fuga, rede para descanso e poleiros. As medidas das gaiolas eram: $120 \mathrm{~cm}$ x $80 \mathrm{~cm}$ x $60 \mathrm{~cm}$. Os doze animais mantinham contato auditivo, visual e olfativo, porém o contato físico só era possível com o seu respectivo parceiro(a).

0 manejo alimentar foi o padronizado pela instituição mantenedora, composto basicamente de frutas da estação, ração para primatas (MegaZoo ${ }^{\circledR}$, MG., Brasil), tenébrios, grilos e ovos, sendo a água ad libitum. Todos os animais deste projeto estavam nas condições descritas por no mínimo 4 anos.

Centro de Reabilitação de Animais Silvestres (CRAS). Os critérios para determinar o estágio do desenvolvimento seguiram aqueles propostos por Yamamoto (1993), onde: infante (0-5 meses), juvenil (5-10 meses), subadulto (10-15 meses) e adultos (>15 meses).

No total das quatro fêmeas que fizeram parte deste estudo, uma tinha seu sistema reprodutor preservado e três estavam laqueadas, conforme informado no Quadro 1.

Quadro 1. Relação dos indivíduos estudados

\begin{tabular}{lcccc}
\hline Indivíduo & Sexo & Casal & Idade & Comentários \\
\hline Anone & Fêmea & Casal 1 & Adulto & Nasceu no CRAS \\
Muriá & Macho & Casal 1 & Adulto & Nasceu no CRAS \\
Sussi & Fêmea/Laqueada & Casal 2 & Adulto & Nasceu no CRAS \\
Clovis & Macho & Casal 2 & Adulto & Origem incerta \\
Thainá & Fêmea/Laqueada & Casal 3 & Adulto & Nasceu no CRAS \\
Mogli & Macho & Casal 3 & Adulto & Origem incerta \\
Maria & Fêmea/Laqueada & Casal 4 & Adulto & Origem incerta \\
Trakinas & Macho & Casal 4 & Adulto & Origem incerta
\end{tabular}




\section{Dados comportamentais}

Os dados comportamentais dos animais foram coletados ao longo de 6 meses, entre maio e novembro de 2010. As observações foram conduzidas pelo método de amostragem focal por intervalo de tempo, com registros de 30 minutos em intervalos de 30 segundos, sendo realizadas sempre no período da manhã (8:00-10:00hs), 5 vezes por semana, totalizando 120 horas ou 14.400 registros para cada animal. Antes de cada período de observação, foi permitido aos animais 20 minutos para que se acostumassem com a presença dos observadores.

Comportamentos sócio-sexuais. Dentre os comportamentos sócio-sexuais observados, sete categorias foram selecionadas como marcadores para o estabelecimento e manutenção do relacionamento social (Evans \& Poole 1983, De Vleeschouwer et al. 2001).

0 repertório sócio-sexual selecionado encontra-se esquematizado na Figura 1 e descrito a seguir:

- "marcação por cheiro": esfregar região genito-anal em um substrato; - "cheirar genitália": encostar ou aproximar o nariz na região ano-genital do outro indivíduo;

- "catação": uso das mãos ou da boca na pelagem de outro indivíduo, para limpeza e remoção de ectoparasitas.
- "aglomeração": dois indivíduos descansarem juntos com pelo menos 30\% da superfície do corpo em contato;

- "apresentação sexual": fêmea ou macho posicionarem-se de maneira quadrupedal, levantando suavemente a base da cauda em direção ao parceiro (a);

- "tentativa de cópula": tentativa pelo macho de segurar a fêmea estando ela em posição quadrupedal, mas não aceitando a cópula;

- "cópula": fêmea em posição quadrupedal enquanto o macho a monta até a obtenção da cópula.

A variável "proximidade entre os animais" foi analisada em separado, sendo apenas uma localização do animal dentro da gaiola em relação ao seu parceiro. 0 critério adotado para esta variável foi considerar o casal como "próximo" quando estavam a uma distância de $30 \mathrm{~cm}$ ou menos e distantes quando esta distância era maior que $30 \mathrm{~cm}$.

\section{Colheita das amostras de fezes}

As amostras de fezes de todos os casais foram colhidas seis vezes por semana no período da manhã, entre 8:00 - 9:00 horas ao longo de seis meses, totalizando 1560 amostras.

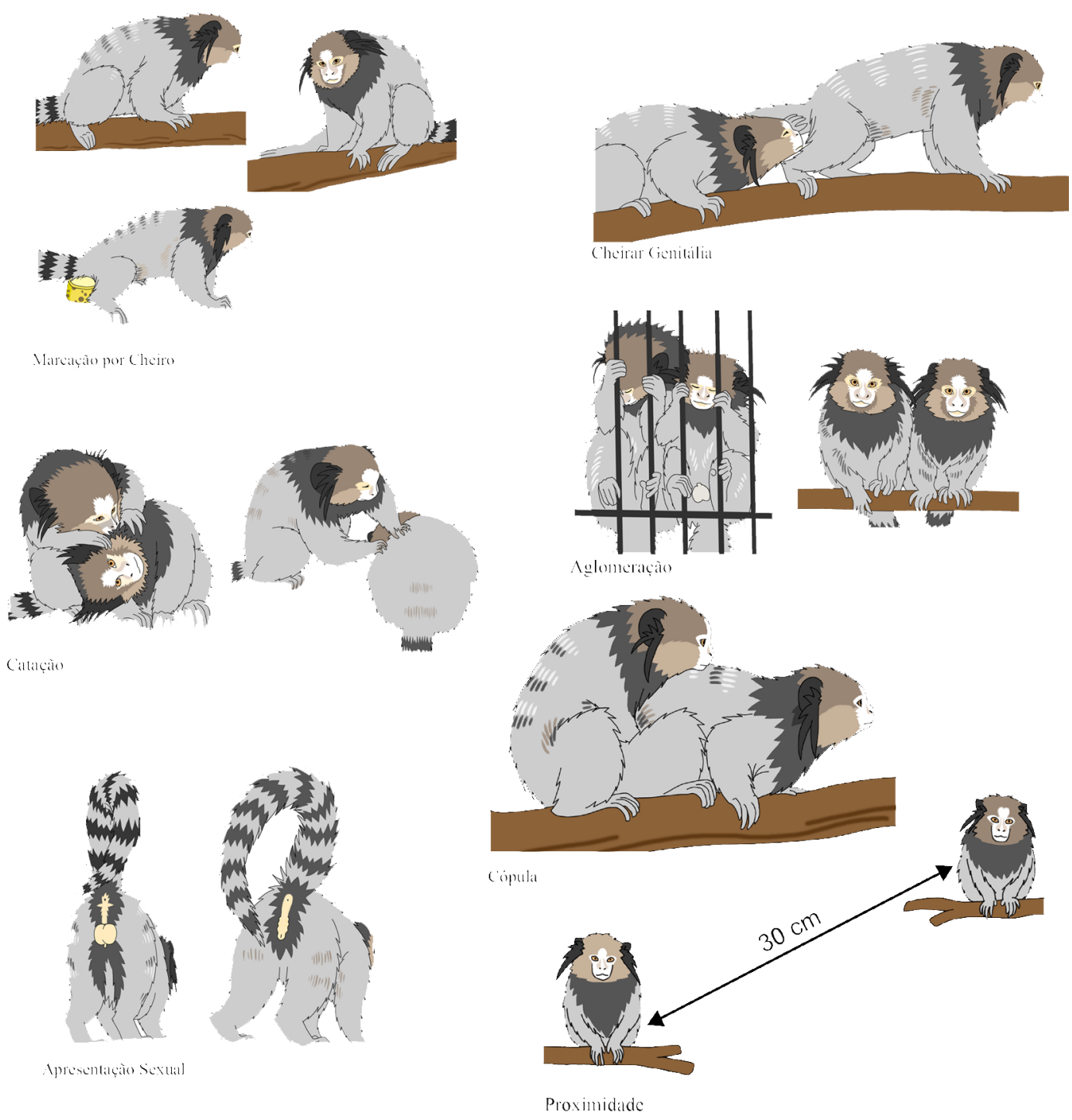

Fig.1. Figuras esquemáticas de comportamentos sócio-sexuais e a variável proximidade entre macho e fêmea de saguis-de-tufo-preto (Callithrix penicillata). 
Para identificação das amostras fecais de machos e fêmeas, os animais receberam todos os dias no período da tarde um suco de frutas com corante alimentício (corante para chocolate Arcolor ${ }^{\circledR}$ ); utilizamos as cores verde ou azul para machos e cor rosa para as fêmeas. As amostras foram homogenizadas e acondicionadas em criotubos identificados com o nome do animal e a data. Todas as amostras foram mantidas em freezer $-20^{\circ}$ até o processamento no Laboratório de Dosagens Hormonais do Departamento de Reprodução Animal da Faculdade de Medicina Veterinária e Zootecnia da Universidade de São Paulo (LDH-VRA-FMVZ/USP).

\section{Extração fecal}

As extrações dos metabólitos fecais foram realizadas segundo técnica preconizada por Ziegler et al (1996) adaptada pelo LDH-FMVZ/USP, conforme brevemente descrita a seguir:

- 0,2g de fezes secas (liofilizadas) foram pesadas e transferidas para tubo de ensaio devidamente identificado;

- Foram acrescidos 5ml de etanol 80\% (Synth, São Paulo, Brasil ); - Os tubos foram colocados em aparelho multivortex (VWR Scientific Products, VX-2500) por 10 minutos, para homogeinização;

- Em seguida, foram centrifugados por 15 minutos a $1500 \mathrm{rpm}$ (Universal 320, Hettich Zentrifugen);

- 1,5ml do sobrenadante (extrato fecal) foi pipetado e transferido para tubo plástico cônico com volume de $2,0 \mathrm{ml}$, identificado com o número da amostra.

- 0 extrato fecal foi armazenado em freezer $-20^{\circ} \mathrm{C}$.

\section{Análises hormonais}

Os metabólitos fecais dos esteroides foram quantificados pela metodologia de enzimaimunoensaio (EIE), utilizando anticorpos monoclonais anti-progesterona CL425 (C. Munro, UC Davis, CA, EUA), HRP progesterona conjugada 3-0-carboxymetiloxime, estradiol, testosterona R 156/7, e microplacas de poliestireno de 96 poços de alta absorção (MaxiSorp, Nunc, Rochester, NY, EUA) as quais foram seladas com um adesivo de acetato. As microplacas foram marcadas ( $50 \mu \mathrm{l} /$ poço) com anticorpo diluído em solução de marcação $\left(\mathrm{Na}_{2} \mathrm{CO}_{3}: 1.59 \mathrm{~g} / \mathrm{L} ; \mathrm{NaHCO}_{3}: 2.93 \mathrm{~g} / \mathrm{L} ; \mathrm{pH}\right.$ 9.6) e incubadas a $4^{\circ} \mathrm{C}$ por $16 \mathrm{hs}$. Após a incubação, as placas foram submetidas a um ciclo de três lavagens com solução de lavagem $(\mathrm{NaCl}$ : 87.66g/L; Tween-20: 0.5\%).

Foram pipetados $150 \mu \mathrm{l}$ de cada extrato fecal e transferidos para tubos de vidro de $15 \mathrm{~mL}$, mantidos em temperatura ambiente para evaporação do álcool. Após a secagem, o material foi ressuspendido com uma solução de hormônio conjugado com enzima (HRP: horseradish peroxidase) diluído em solução tampão $\left(\mathrm{NaH}_{2} \mathrm{PO}_{4} \cdot \mathrm{H}_{2} \mathrm{O}: 5.421 \mathrm{~g} / \mathrm{L} ; \mathrm{Na}_{2} \mathrm{HPO}_{4}: 8.662 \mathrm{~g} / \mathrm{L} ; \mathrm{NaCl}: 8.7 \mathrm{~g} / \mathrm{L} ; \mathrm{Albu}-\right.$ mina sérica bovina: $1.0 \mathrm{~g} / \mathrm{L}$; pH 7.0).

Foram adicionados $50 \mu \mathrm{l}$ de solução tampão em cada poço e posteriormente $50 \mu \mathrm{l}$ de cada padrão da curva $(0.1-10,000 \mathrm{pg} /$ $\mathrm{mL}$ ), dos controles ou da solução de amostra com HRP. As microplacas foram seladas e incubadas por 2 horas em temperatura ambiente. Após a incubação, as microplacas foram lavadas, secas e posteriormente adicionado $100 \mu \mathrm{l}$ /poço de substrato cromógeno (TMB substrato, Neogen do Brasil). A reação cromógena foi parada com $100 \mu$ l de solução STOP $\left(\mathrm{H}_{2} \mathrm{SO}_{4} 96 \%\right.$ : 10\%). A densidade óptica foi medida em uma leitora automática (DTX 800 Multimode Detector, Beckman Coulter, Brea, CA, EUA) utilizando o filtro de $450 \mathrm{~nm}$.

Todas as amostras foram analisadas em duplicata. As diluições utilizadas foram de 1:8.000, 1:10.000 e 1:10.000 para os anticorpos CL425, R0008 e R156/7, respectivamente. Já para os HRPs, foram de 1:160.000, 1:160.000 e 1:40.000 para os hormônios progesterona, estradiol e testosterona, respectivamente.

Foram realizados os controles de qualidade dos ensaios com o cálculo dos coeficientes de variação altos e baixos, intra e inter ensaios.

\section{Critérios para determinação das fases do ciclo ovariano}

Para a caracterização das fases do ciclo ovariano foram utilizados os resultados das mensurações das concentrações de metabólitos fecais de progesterona. All samples above the mean plus 2 standard deviations (SD) were removed. The mean was recalculated, and this process was repeated until all values were within 2 SD from the mean. Outliers (2 SD above the mean) were removed to reduce the variability, so that the remaining values were most similar to each other. The resulting mean value was referred to as the "baseline" and all values greater than the mean plus 2,0 SD were considered "peak." This process allows for a statistical assessment of baseline values for biologically relevant hormone concentrations (Heintz et al. 2011). Para a determinação do fim de uma fase folicular e início de uma fase lútea, foi considerada a ocorrência de dois ou mais valores sequenciais acima dos níveis basais que se mantivessem de forma sustentada, sinalizando a ocorrência de ovulação. 0 fim da fase lútea foi determinado com o retorno de dois ou mais valores sequenciais de concentrações aos níveis considerados basais.

Uma terceira fase foi descrita como período periovulatório, sendo definida como o período compreendido entre as 24 horas antes (d-1) e 24 horas depois $(\mathrm{d}+1)$ do dia estimado da ovulação (d 0), caracterizado este por sua vez, pelo início do aumento das concentrações sustentadas de metabólitos fecais de progesterona (Converse et al. 1995).

\section{Análise estatística}

Através do aplicativo Guided Data Analisys, os dados foram testados quanto à normalidade dos resíduos (distribuição normal) e homogeneidade das variâncias.

Para cada uma das fases do ciclo ovariano (folicular, periovulatória e lútea) foram calculadas as médias e erros padrão das medias, das concentrações de metabolitos fecais de progesterone e de estrógenos para as fêmeas e de metabolitos fecais de testosterona para os machos. As médias foram comparadas entre si com o uso do teste ANOVA, complementada pelo Teste de Tukey, ambos com $\mathrm{p}<0.05$.

Os registros comportamentais foram analisados por frequência de ocorrência (\%) para cada fase do ciclo ovariano e comparados entre si utilizando o Fisher's exact test, considerando significativo o $\mathrm{p}<0.05$.

Este trabalho foi aprovado pela Comissão de Bioética da Faculdade de Medicina Veterinária e Zootecnia, Universidade de São Paulo, Brasil, sob o processo número 1470/2008.

\section{RESULTADOS}

Os ensaios hormonais apresentaram coeficientes de variação FMP (inter ensaio baixo: 4.3\%, alto: 5.4\%; intra ensaio baixo: $3.9 \%$; alto: $5.6 \%$ ), FME (inter ensaio baixo: 0.5\%, alto: 2.8\%; intra ensaio baixo: $4.3 \%$; alto: $5.7 \%$ ) e FMT (inter ensaio baixo: $5.8 \%$, alto: $10.1 \%$; intra ensaio baixo: $6.3 \%$; alto: 8.6\%). A sensibilidade dos ensaios de FMP, FME e FMT foram $1.50 \mathrm{pg} / \mathrm{ml}, 1.52 \mathrm{pg} / \mathrm{ml}$ e $1.02 \mathrm{pg} / \mathrm{ml}$ respectivamente.

A análise das variações das concentrações de metabólitos fecais de progesterona indicou a ocorrência no total de 31 ciclos ovarianos completos no período de 6 meses. Todas as fêmeas apresentaram ciclos ovarianos ovulatórios no período. A duração média do ciclo ovariano foi de $24.3 \pm 4.1$ dias, sendo que a fase folicular durou $13.04 \pm 4.8$ dias e a fase lútea 11.2 \pm 4.2 dias. 
Quadro 2. Média ( \pm erro padrão da média) das concentrações de metabólitos fecais de progesterona, estradiol de fêmeas e testosterona de machos durante o ciclo ovariano de fêmeas de saguis-de-tufo-preto (Callithrix penicillata), mantidos em cativeiro, São Paulo 2012

\begin{tabular}{lccc}
\hline & $\begin{array}{c}\text { Fase } \\
\text { folicular }\end{array}$ & $\begin{array}{c}\text { Fase } \\
\text { periovulatória }\end{array}$ & $\begin{array}{c}\text { Fase } \\
\text { lútea }\end{array}$ \\
\hline $\begin{array}{l}\text { MFP - fêmeas } \\
\text { (ng/g) } \pm \text { DP }\end{array}$ & $1873.00^{\mathrm{a}} \pm 32.75$ & $4808.15^{\mathrm{b}} \pm 11.39$ & $10177.63^{\mathrm{c}} \pm 171.71$ \\
$\begin{array}{l}\text { MFE - fêmeas } \\
\text { (ng/g) } \pm \text { DP }\end{array}$ & $490.01^{\mathrm{a}} \pm 96.07$ & $269.38^{\mathrm{b}} \pm 80.64$ & $1068.63^{\mathrm{c}} \pm 196.70$ \\
$\begin{array}{l}\text { MFT - machos } \\
\text { (ng/g) } \pm \text { DP }\end{array}$ & $148.17^{\mathrm{a}} \pm 15.5$ & $155.92^{\mathrm{a}} \pm 18.38$ & $156.20^{\mathrm{a}} \pm 12.05$ \\
\end{tabular}

$\overline{\mathrm{a}, \mathrm{b}, \mathrm{c}}$ Letras diferentes sobrescritas na mesma linha indicam diferença estatística significativa $(\mathrm{P}<0.05)$.

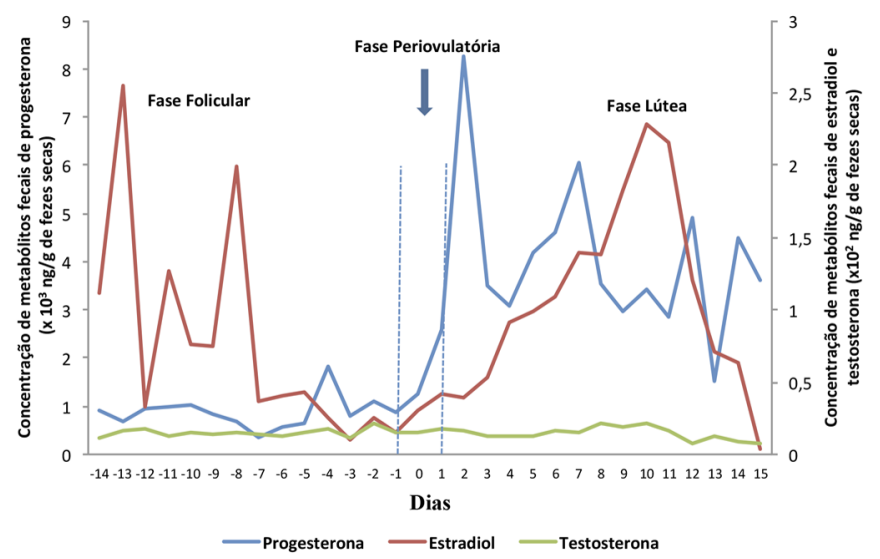

Fig.2. Média das concentrações de metabólitos fecais de progesterona, estradiol de fêmeas e testosterona de machos durante o ciclo ovariano de fêmeas de saguis-de-tufo-preto (Callithrix penicillata), São Paulo 2012.

Nas diferentes fases do ciclo ovariano (fase folicular, lútea e peri-ovulatória) foram observadas variações nas concentrações dos metabólitos fecais (Quadro 2, Fig.2).

Os comportamentos sócio sexuais e a proximidade entre os animais mostraram-se mais evidentes na fase periovulatória, quando comparado às demais fases do ciclo ovariano (Quadro 3, Fig.3 e 4).

\section{DISCUSSÃO}

Na literatura existem poucas informações endócrino reprodutivas para primatas da família Callitrichidae (Lottker et al. 2004, French et al. 1992, Gilchrist et al. 2001, Tardif et al. 2003), sendo que apenas duas espécies do gênero Callithrix (C. jacchus e C. kuhlii) foram adequadamente estudadas (Mustoe et al. 2012). Este é o primeiro estudo endócrino reprodutivo e do comportamento sócio-sexual de sagui-de-tufos-pretos (Callithrix penicillata), mantidos em cativeiro. Os resultados das concentrações de MFP possibilitaram a determinação da duração do ciclo ovariano e a caracterização endócrina das três fases que o compõem (folicular, periovulatória e lútea). A média encontrada de $24,3 \pm 4,1$ dias para a duração do ciclo ovariano é semelhante à descrita para Callithrix geoffroyi 27.82 \pm 1.49 dias, C. kuhlii 25 dias (French et al. 1996) e C. jacchus 27,7 dias (Kendrick \& Di-
Quadro 3. Frequência de ocorrência (\%) dos comportamentos sócio-sexuais e da proximidade em casais de saguis-de-tufo-preto (Callithrix penicillata), mantidos em cativeiro. São Paulo, 2012

\begin{tabular}{lccc}
\hline & Fase folicular & Fase periovulatória & Fase lútea \\
\hline Proximidade & $65,79^{\mathrm{a}}$ & $97,33^{\mathrm{b}}$ & $64,8^{\mathrm{a}}$ \\
Marcação por cheiro & $0,61^{\mathrm{a}}$ & $1,62^{\mathrm{b}}$ & $0,59^{\mathrm{a}}$ \\
Cheirar genitália & $0,04^{\mathrm{a}}$ & $7,82^{\mathrm{b}}$ & $0,03^{\mathrm{a}}$ \\
Catação & $1,19^{\mathrm{a}}$ & $9,82^{\mathrm{b}}$ & $0,96^{\mathrm{a}}$ \\
Aglomeração & $0,33^{\mathrm{a}}$ & $1,02^{\mathrm{b}}$ & $0,29^{\mathrm{a}}$ \\
Apresentação Sexual & $0,01^{\mathrm{a}}$ & $0,16^{\mathrm{b}}$ & $0,02^{\mathrm{a}}$ \\
Tentativa de cópula & 0 & 0,05 & 0,01 \\
Cópula & $0,22^{\mathrm{a}}$ & $0,83^{\mathrm{b}}$ & $0,09^{\mathrm{a}}$
\end{tabular}

$\overline{a, b, c}$ Letras diferentes sobrescritas nas linhas indicam diferença estatística significativa $(\mathrm{P}<0.05)$.

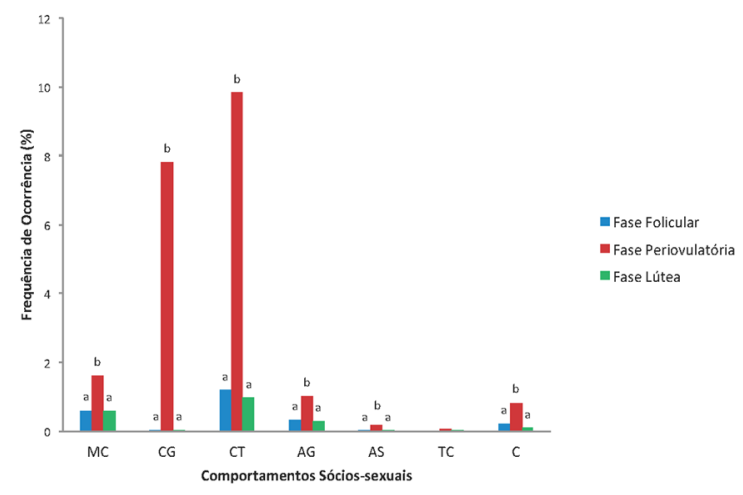

MC: Marcação por cheiro; CG: Cheirar genitália; CT: Catação; AG: Aglomeração; AS: Apresentação Sexual; TC: Tentativa de cópula; C: Cópula

Fig.3. Frequência de ocorrência (\%) de comportamentos sócio-sexuais entre o macho e a fêmea de sagui-de-tufo-preto (Callithrix penicillata) durante as fases do ciclo ovariano, São Paulo, 2012.

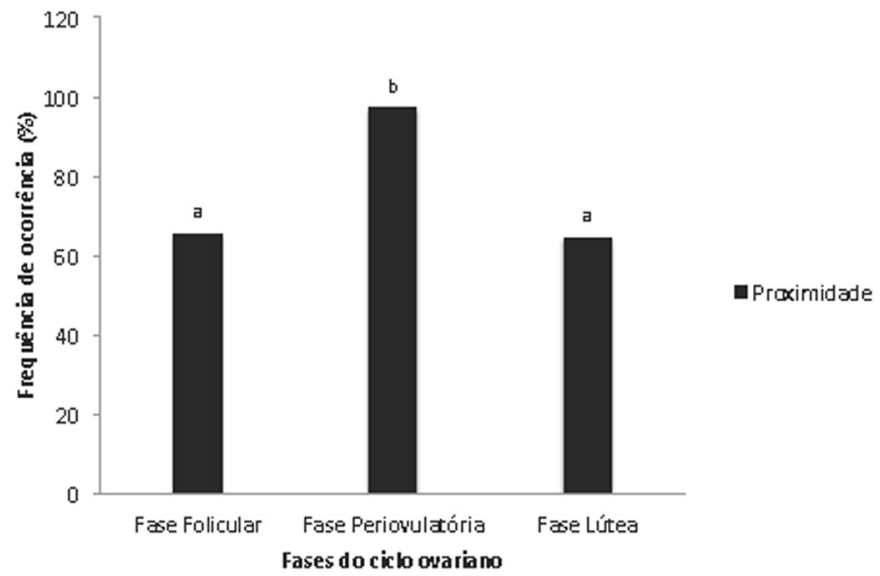

Fig.4. Frequência de ocorrência (\%) de proximidade entre o macho e a fêmea de sagui-de-tufo-preto (Callithrix penicillata) durante as fases do ciclo ovariano. São Paulo, 2012.

xson 1983, Gilchrist 2001, Dixson 2013). As fases folicular e lútea apresentaram duração de $13.04 \pm 4.8$ e de $11.2 \pm 4.2$ dias, respectivamente.

Muitas espécies não apresentam alterações morfológicas visualmente perceptíveis na fase periovulatória, sendo 
que a maior ocorrência de comportamentos sócio sexuais nesta fase pode ser interpretada como um sinalizador do período fértil (Ziegler 2013). A análise simultânea dos padrões comportamentais sócio sexuais e das variações das concentrações de metabólitos hormonais, permitiu uma melhor caracterização das fases. Neste caso, os resultados mostraram aumento significativo das concentrações de metabólitos fecais de progesterona concomitante com o aumento do repertório comportamental sócio sexual entre os casais na fase periovulatória, quando comparados aos das outras duas fases.

A análise conjunta endócrino-comportamental também foi descrita em Callithrichidae por Ziegler (1987), que mostrou a ocorrência da concepção em mais de $85 \%$ das ovulações, sugerindo a existência de algum tipo de sinalização das fêmeas sobre o seu "status" ovulatório. Esta comunicação do momento da ovulação associada à ocorrência do comportamento sexual, são importantes para garantir a fertilização bem sucedida (Ziegler 2013).

Em Callithrichidae, as cópulas tem sido observadas ao longo de todo o ciclo ovariano (Dixon 2013), dificultando, do ponto de vista comportamental, a identificação do momento da ovulação. Para Kendrick \& Dixon (1983), as cópulas ficam mais frequentes na fase folicular, atingindo seu ápice, na fase periovulatoria, quando o tongue-flicking também mostra-se mais evidente. Neste trabalho foi possível confirmar o aumento de frequência das cópulas a partir da fase folicular e evidenciar significativamente seu aumento na fase periovulatoria, porém, sem nenhuma correlação com o tongue-flicking.

O tongue-flicking é um comportamento sócio-sexual importante e relevante na fase periovulaória, descrito em C. jacchus (Dixson 2001, 2013), porém, este comportamento não foi observado em momento algum neste trabalho e portanto, não foi considerado como um dos sinalizadores de comportamento sócio-sexual.

Já em relação à proximidade e à catação, nossos resultados mostram aumento significativo destas duas variáveis na fase periovulatória. A estimulação tátil entre dois indivíduos assinala uma relação espacial que pode promover um sucesso na reprodução, uma vez que a catação fortalece os laços sociais e aumenta as chances de cópula (Tutin 1979, Ziegler 2013). Na fase periovulatória, foi também observada de forma significativa (Quadro 3) a apresentação da genitália, tanto de fêmeas para machos, quanto de machos para fêmeas. Este comportamento tem sido descrito para outros primatas como proceptivo (Strier 2011).

Os saguis de maneira geral são animais extremamente sensíveis aos estímulos sensoriais, seja pela sua forma tátil (Tutin 1979) ou pela olfativa (Smith \& Abbott 1998, Ziegler 2013). Machos e fêmeas possuem glândulas especializadas na região anogenital (Epple 1981, 1982). Nas fêmeas, a secreção destas glândulas associada à urina, fezes e secreções genitais pode sinalizar olfativamente o seu período fértil (Smith \& Abbott 1998, Ziegler et al. 1993, Smith \& Abbott 1998). Nossos resultados demonstraram aumento significativo dos comportamentos de "cheirar genitália" e da "marcação por cheiro" na fase periovulatória (Quadro 3 ), sugerindo que este comportamento pode ser de fato um sinalizador importante do momento da ovulação em $C$. penicilatta. Estas sinalizações odoríferas parecem ser muito importantes para esta espécie, uma vez que os Callithrichidae, em geral, são animais que compartilham de um sistema de reprodução cooperativa, onde todos os membros da mesma família se mantêm unidos para garantir o êxito de todo o grupo (Ziegler 2013). Além disto, a liberação de odores sinalizando o momento da ovulação pode ser relevante para pequenas espécies de primatas arborícolas, pois sinais visuais podem não ser percebidos e os auditivos (vocalizações) podem ser confundidos com sons de pássaros. As "marcas de cheiro" podem permanecer por longos períodos nos substratos, o que pode torná-las mais eficientes (Ziegler 2013).

Apesar de haver aumento das atividades sócio-sexuais na fase periovulatória, não foi detectado aumento nas concentrações de metabólitos fecais de testosterona nos machos ao longo de todo o ciclo ovariano das fêmeas.

\section{CONCLUSÕES}

Os resultados destes estudos permitiram descrever, do ponto de vista endócrino, o ciclo ovariano e suas fases em Callithrix penicillata, utilizando a extração e dosagem de metabólitos fecais de esteróides sexuais.

A análise conjunta das concentrações de metabólitos fecais de esteróides sexuais e dos comportamentos sócio-sexuais possibilitou uma melhor caraterização das relações endócrino-comportamentais de $C$. penicillata.

O sagui-de-tufo-preto apresenta características endócrinas sexuais semelhantes às outras espécies de Callitrhichideos, porém difere na ocorrência dos comportamentos sócio-sexuais.

Agradecimentos.- Os autores gostariam de agradecer à FAPESP (Fundação de Amparo à Pesquisa do Estado de São Paulo) pelo suporte financeiro (10/51926-2 e 08/53562-8) e à Cintya Eimy Kato, veterinária e ilustradora, autora dos desenhos esquemáticos deste trabalho.

\section{REFERÊNCIAS}

Beck B.B., Anderson D., Ogden J., Rettberg B., Brejla C., Scola R. \& Warneke M. 1982. Breeding the Goeldi's monkey Callimico goeldii at Brookfield Zoo, Chicago. Intl Zoo Ybk 22:106-114.

Carosi M., Heistermann M. \& Visalberghi E. 1999. The display of proceptive behaviors in relation to urinary and fecal progestin levels over the ovarian cycle in female tufted capuchin monkeys. Horm. Behav. 36:252-265.

Carroll J.B. 1982. Maintenance of the Goeldi's monkey at Jersey Wildlife Preservation Trust. Intl Zoo Ybk . 22(1):101-105.

Converse L.J., Carlson A., Ziegler T.E. \& Snowdon C.T. 1995. Communication of ovulatory state to mates by female pygmy marmosets, Cebuella pygmaea. Anim. Behav. 49:615-621.

De Vleeschouwer K., Van Elsacker L. \& Leus K. 2001. Multiple breeding females in captive groups of golden-headed lion tamarins (Leontopithecus chrysomelas): causes and consequences. Folia Primatol. 72:1-10.

Dixson A.F. 1990. Medial hypothalamic lesions and sexual receptivity in the female common marmoset (Callithrix jacchus). Folia Primatol. 54:46-56.

Dixson A. 2001. The evolution of neuroendocrine mechanisms regulating sexual behaviour in female primates. Reprod. Fertil. Dev. 13:599-607.

Dixson A.F. 2013. Primate Sexuality: comparative studies of the prosimians, monkeys, apes and human beings. Oxford University Press, Oxford. 785p. 
Epple G. 1981. Effects of prepubertal castration on the development of the scent glands, scent marking, and aggression in the saddle back tamarin (Saguinus fuscicollis, Callitrichidae, Primates). Horm. Behav. 15:54-67.

Epple G. 1982. Effects of prepubertal ovariectomy on the development of scent glands, scent marking, and aggressive behaviors of female tamarin monkeys (Saguinus fuscicollis). Horm. Behav. 16:330-342.

Evans S. \& Poole T.B. 1983. Pair-bond formation and breeding success in the common marmoset Callithrix jacchus jacchus. Int. J. Primatol. 4(1):83-97.

Faria D.S. 1989. 0 grupo social em Callithrix penicillata, o mico-estrela do Planalto Central Brasileiro: Estudo realizado na floresta de galeria do córrego Capetinga, Brasília. Tese de Doutorado, Universidade de São Paulo, São Paulo.

Fedigan L.M. 1982. Primate Paradigms: sex roles and social bonds. Eden Press, Montreal, Canada. 101p.

French J.A., DeGraw W.A., Hendricks S.E., Wegner F. \& Bridson W.E. 1992. Urinary and plasma gonadotropin concentrations in golden lion tamarins (Leontopithecus r. rosalia). Am. J. Primatol. 26:53-59.

French J.A., Brewer K.J., Schaffner C.M., Schalley J., Hightower-Merritt D., Smith T.E. \& Bell S.M. 1996. Urinary steroid and gonadotropin excretion across the reproductive cycle in female Wied's black tuftedear marmosets (Callithrix kuhlii). Am. J. Primatol. 40:231-245.

Gilchrist R.B., Wicherek M., Heistermann M., Nayudu P.L. \& Hodges J.K. 2001. Changes in follicle-stimulating hormone and follicle populations during the ovarian cycle of the com- mon marmoset. Biol. Reprod. 64:127-135.

Heintz M.R., Satymire R.M., Parr L.A. \& Lonsdorf E.V. 2011. Validation of a cortisol enzyme Immunoassay and characterization of salivary cortisol circadian rhythm in chimpanzees (Pan troglodytes). Am. J. Primatol. 73:903-908.

Higham J.P., Heistermann M., Saggau C., Agil M., Perwitasari-Farajallah D. \& Engelhardt A. 2012. Sexual signalling in female crested macaques and the evolution of primate fertility signals. Evol. Biol. 12:89.

Kendrick K.M. \& Dixson A.F. 1983. The effect of the ovarian cycle on the sexual behav- ior of the common marmoset (Callithrix jacchus). Physiol. Behav. 30:735-742.

Lottker P., Huck M., Heymann E.W. \& Heistermann M. 2004. Endocrine correlates of reproductive status in breeding and nonbreeding wild female moustached tamarins. Int. J. Primatol. 25:919-937.

Lindburg D.G., Beckson J. \& Nightenhelser L. 1986. The contributions of zoos to primate conservation, p.295-300. In: Else J.G. \& Lee P.C. (Eds), Primate Eology and Conservation. Cambridge University Press, Cambridge.
Mustoe A.C., Jensen H.A. \& French J.A. 2012. Describing Ovarian Cycles, Pregnancy Characteristics, and the Use of Contraception in Female White-Faced Marmosets, Callithrix geoffroyi. Am. J. Primatol. 74:10441053.

Rimbach R., Heymann E.W., Link A. \& Heistermann M. 2013. Validation of an enzyme immunoassay for assessing adrenocortical activity and evaluation of factors that affect levels of fecal glucocorticoid metabolites in two New World primates. Gen. Comp. Endocrinol. 191:13-23.

Smith T.E. \& Abbott D.H. 1998. Behavioral discrimination be- tween circumgenital odor from peri-ovulatory dominant and anovulatory female common marmosets (Callithrix jacchus). Am. J Primatol. 46:265-284.

Stevenson M.F. \& Rylands A.B. 1988. The marmosets, genus Callithrix, p.131-222. In: Mittermier R.A., Rylands A.B., Coimbra-Filho A. \& Fonseca G.A.B. (Eds), Ecology end Behavior of Neotropical Primates. World Wildlife Fund, Washington, DC.

Strier K.B. 2011. Primate Behavioral Ecology. 4th ed. Prentice Hall, Boston.

Tardif S.D., Smucny D.A., Abbott D.H., Mansfield K., Schultz-Darken N. \& Yamamoto M.E. 2003. Reproduction in captive common marmosets (Callithrix jacchus). Comp. Med. 53:364-368.

Tutin G. 1979. Mating patterns and reproductive strategies in a community of wild chimpanzees (Pantroglodytes schweinfurthii). Behav. Ecol. Sociobiol. 6:29-38.

Yamamoto M.E. 1993. From dependence to sexual maturity: the behavioral ontogeny of Callitrichidae, p.235-254. In: Rylands A.B. (Ed.), Marmosets and Tamarins: systematics, behaviour and ecology. Oxford University Press, Oxford.

Ziegler T.E., Savage A., Schelfer G. \& Snowdon C.T. 1987. The endocrinology of puberty and reproductive functioning in cotton-top tamarins (Saguinus oedipus) under varying social conditions. Biol. Reprod. 37:618-627.

Ziegler T.E., Epple G., Snowdon C.T., Porter T.A., Belcher A.M. \& Küderling I. 1993. Detection of the chemical signals of ovulation in the cotton top tamarin, Saguinus oedipus. Anim. Behav. 45:313-322.

Ziegler T.E., Scheffler G., Wittwer D.J., Schultz-Darken N., Snowdon C.T. \& Abbott D.H. 1996. Metabolism of reproductive steroids during the ovarian cycle in two species of Callitrichids, Saguinus Oedipus and Callithrix jacchus, and estimation of the ovulatory period from fecal steroids. Biol. Reprod. 54:91-99.

Ziegler T.E. 2013. Social effects via olfactory sensory stimuli on reproductive function and dysfunction in cooperative breeding marmosets and tamarins. Am. J. Primatol. 75:202-211. 